\title{
Minimally Knotted Embeddings of Planar Graphs
}

\author{
Ying-Qing $\mathrm{Wu}$
}

A graph $\Gamma$ is planar if it can be embedded into a 2 -sphere. An embedding $\varphi$ of $\Gamma$ into 3 -sphere $S^{3}$ is planar or unknotted if there is a 2-sphere in $S^{3}$ containing $\varphi(\Gamma)$. Otherwise it is knotted. An embedding $\varphi$ of $\Gamma$ into $S^{3}$ is called minimally knotted if $\varphi(\Gamma)$ is knotted, but all proper subgraphs of $\varphi(\Gamma)$ are unknotted. A free edge of $\Gamma$ is an edge $e$ such that $e$ has distinct endpoints, and one of its endpoints is incident to no other edges of $\Gamma$. Clearly, if $\Gamma$ has a free edge, or if it has an isolated vertex, then it has no minimally knotted embeddings. It was conjectured [2,3] that all other planar graphs admit minimally knotted embeddings. A proof of this conjecture was given by Kawauchi in [1], using "hyperbolic imitations".

Theorem 1 If $\Gamma$ is a planar graph with no free edges or isolated vertices, then it admits a minimally knotted embedding into $S^{3}$.

Notice that the graph in the theorem is not necessarily connected. From the proof it is clear that there are actually infinitely many minimally knotted embeddings. Some special cases have also been proved by Simon and Wolcott [3]. The present note is to give an alternative proof of this theorem, which seems simpler and more natural. The idea is to consider some surfaces in the graph exterior, and show that they are incompressible. It then follows that the graph is unknotted only if each manifold piece obtained by cutting the graph exterior along these surfaces has free fundermental group. This will reduce the knottedness of the embedding to a "local" problem.

We need some basic facts about decomposable graphs. A graph $G$ in $S^{3}$ is called decomposable if there is a 2-sphere $S$ in $S^{3}$ such that $S \cap G$ is a vertex $v_{0}$ of $G$, and both sides of $S$ contain edges of $G$ incident to $v_{0}$. We call $v_{0}$ a decomposing vertex, and $S$ a decomposing sphere of $G$. Choose a regular neighborhood $N(v)$ for each vertex $v$ of $G$, and choose a regular neighborhood $N(e)$ for each edge, so that the disks $N(e) \cap \partial N(v)$ are 
mutually disjoint. Then $N(G)=(\cup N(e)) \cup(\cup N(v))$ is a regular neighborhood of $G$ in $S^{3}$. Denote by $E(G)=S^{3}-\operatorname{Int} N(G)$ the exterior of $G$, and by $\delta\left(v_{0}\right)$ the punctured sphere $\partial N\left(v_{0}\right)-\operatorname{Int} N(G)$ on the boundary of $E(G)$. A decomposing sphere of $\Gamma$ at $v_{0}$ gives rise to a compressing disk of $\delta\left(v_{0}\right)$ in $E(G)$. Conversely, any compressing disk of $\delta\left(v_{0}\right)$ can be extended to a decomposing sphere of $\Gamma$ at $v_{0}$. Therefore we have the following

Lemma $1 v_{0}$ is a decomposing vertex if and only if $\delta\left(v_{0}\right)$ is compressible in $E(G)$.

Now suppose $e_{1}, e_{2}$ are two edges of $G$. They are called parallel if there is a map $\varphi: D^{2} \rightarrow S^{3}$ such that $\varphi\left(\partial D^{2}\right)=e_{1} \cup e_{2}$, and $\left.\varphi\right|_{\operatorname{Int} D^{2}}$ is an embedding of $\operatorname{Int} D^{2}$ into $S^{3}-G$. A subgraph $H$ of $G$ is a reduction of $G$ if $G-H$ consists of some edges, each of which is parallel to some edge in $H$. We say that $H$ is obtained from $G$ by removing some parallel edges. An edge of $G$ is a cycle if its two endpoints are on the same vertex.

Lemma 2 Let $H$ be a reduction of a graph $G$, and suppose every component of $G$ has at least 2 vertices. If $G$ is decomposable, then $H$ is also decomposable.

Proof. By induction we need only to prove the case that $H$ is obtained from $G$ by removing a single edge $e^{\prime}$ which is parallel to some edge $e^{\prime \prime}$. Let $S$ be a decomposing sphere of $G$, and let $B_{1}, B_{2}$ be the 3-balls with $B_{1} \cap B_{2}=S$. Suppose $v_{0}=S \cap G$. By definition, there are edges $e_{i}$ in $B_{i}$ incident to $v_{0}$. Since any component has at least two vertices, we can choose $e_{2}$, say, to be a noncycle edge. If $e^{\prime} \neq e_{i},(i=1,2)$, then it is clear that after removing $e^{\prime}$ the sphere $S$ is still a decomposition sphere, and we are done. So suppose $e^{\prime}=e_{1}$. (The other case is similar). Since $e^{\prime \prime}$ is parallel to $e^{\prime}$, by an isotopy of $e^{\prime \prime}$ we may assume that $e^{\prime \prime}$ also lies in $B_{1}$. Now $e_{1}$ and $e_{2}$ are not parallel because $e_{2}$ must have an endpoint in the interior of $B_{2}$, while $e_{1}$ has both endpoints in $B_{1}$. Thus $e_{2} \neq e^{\prime \prime}$. The two edges $e_{2}$ and $e^{\prime \prime}$ of $H$ lie on different sides of $S$, therefore $S$ is a decomposing sphere of $H$.

Lemma 3 Let e be a cycle edge of $G$ bounding an embedded disk $D$ in $S^{3}$ so that Int $D$ intersects $G$ transversely at a single point $v$ on some edge $e^{\prime}$. Let $H$ be the graph obtained from $G \cup D$ by shrinking $D$ into a single vertex. If $G$ is decomposable, then so is $H$.

Proof. Let $S$ be a decomposing sphere of $G$, intersecting $G$ at a vertex $v_{0}$, isotoped so that $S \cap D$ is a union of some circles, and different circles intersect only possibly at $v^{\prime}=\partial e$. We choose $S$ so that the number of circles in $S \cap D$ is minimal. 
Suppose $S \cap \operatorname{Int} D \neq \emptyset$. Let $C$ be a circle of $S \cap D$ which is innermost on the sphere $S$, i.e. it bounds a disk $D^{\prime}$ in $S$ with interior disjoint from $D$. Denote by $D^{\prime \prime}$ the disk bounded by $C$ in $D$.

If $D^{\prime \prime}$ intersects $e^{\prime}$, then after shrinking $D$ to a point $D^{\prime} \cup D^{\prime \prime}$ becomes a sphere $\bar{S}$ such that the two edges of $H$ coming from $e^{\prime}$ are on different sides of $\bar{S}$, so $\bar{S}$ is a decomposing sphere of $H$ in $S^{3}$, and the result follows. If $D^{\prime \prime}$ does not intersect $e^{\prime}$, then $D^{\prime} \cup D^{\prime \prime}=S^{\prime}$ is a sphere in $S^{3}$ which is either disjoint from $G$ or intersects $G$ at $v_{0}$. We get contradictions as follows:

If on both sides of $S^{\prime}$ there are edges incident to $v_{0}$, then $S^{\prime}$ is a decomposing sphere of $G$ which can be isotoped off the interior of $D$, contradicting the choice of $S$. So we assume $S^{\prime}$ bounds a 3 -ball $B$ such that no edges of $G$ in $B$ is incident to $v_{0}$. Using $B$ one can find an isotopy of $S$ in $S^{3}$ which fixes $v_{0}$ and pushes the rest of $S \cap B$ (including $D^{\prime}$ ) out of $B$ through the disk $D^{\prime \prime}$, resulting in a sphere $\bar{S}$ whose intersection with $D$ has fewer circles than $S$ does. This isotopy may intersect the components of $G$ lying in $B$. But since none of these components has edges incident to $v_{0}$, any two edges of $G$ lying on different sides of $S$ are still on different sides of $\bar{S}$, so $\bar{S}$ is a decomposing sphere of $G$. It contradicts the minimality of the number of circles in $S \cap D$.

We now suppose $S \cap \operatorname{Int} D=\emptyset$. If $S$ is also disjoint from $v^{\prime}=\partial e$, then $S \cap D=\emptyset$, and it is obvious that $S$ remains a decomposing sphere of $H$. If $S$ intersects $v^{\prime}$, then $v^{\prime}=v_{0}=S \cap G$. Let $B_{1}, B_{2}$ be the 3-balls such that $B_{1} \cap B_{2}=S$, and suppose $D$ lies in $B_{1}$. By assumption there are edges of $G$ in $B_{2}$ which are incident to $v_{0}$. These edges are also edges of $H$. After shrinking $D$ into a point, the edge $e^{\prime}$ becomes two edges, both lie in $B_{1}$ and are incident to $v_{0}$. Since on both sides of $S$ there are edges of $H$ incident to $v_{0}$, $S$ is a decomposing sphere of $H$.

An $n$-strand tangle is a pair $(B, A)$, where $B=D^{2} \times I$ is a 3 -ball, and $A$ is the union of $n$ properly embedded, mutually disjoint arcs with $\partial A \subset \partial D^{2} \times\{1 / 2\}$. Usually we present a diagram of a tangle by drawing the graph $A \cup\left(\partial D^{2} \times\{1 / 2\}\right)$ so that $\partial D^{2} \times\{1 / 2\}$ is the outside circle of the diagram. Two tangles $(B, A)$ and $\left(B, A^{\prime}\right)$ are equivalent if $A$ is isotopic to $A^{\prime}$ rel $\partial A$. A tangle $(B, A)$ is trivial if it has a diagram with no crossings, i.e. $A$ can be isotoped (rel $\partial A$ ) to lie in $D^{2} \times\{1 / 2\}$.

Let $T_{n}$ be a tangle $(B, A)$, where $A$ consists of $2^{n}$ parallel arcs, each of which is a "trefoil" $\operatorname{arc}$ in $B$, i.e. an arc whose exterior in $B$ is homeomorphic to the trefoil knot 
exterior in $S^{3}$. See Figure 1 for a diagram of $T_{2}$. Let $M_{n}=B-\operatorname{Int} N(A)$, and let $F_{n}$ be the punctured sphere $\partial B \cap M_{n}$.

(Figure 1)

Lemma $4 F_{n}$ is incompressible in $M_{n}$, and $\pi_{1} M_{n}$ is not a free group.

Proof. Let $X$ be the exterior of a trefoil knot in $S^{3}$, let $Y=P_{n} \times I$, where $P_{n}$ is a disk with $2^{n}$ holes, and $I$ is the unit interval. Denote by $C$ the annulus (outer circle of $P_{n}$ ) $\times I$. Then $M_{n}$ is homeomorphic to $X \cup_{C} Y$, where $C$ is identified with a regular neighborhood of a meridian on $\partial X$. By an innermost circle outermost arc argument it can be shown that any compressing disk of $F_{n}$ in $M_{n}$ can be isotoped off $C$, so it lies in either $X$ or $Y$. But clearly $F_{n} \cap X$ and $F_{n} \cap Y$ are both incompressible. Therefore $F_{n}$ is incompressible in $M_{n}$. Also, since $C$ is incompressible in $M_{n}$, the trefoil knot group $\pi_{1} X$ is a subgroup of $\pi_{1} M_{n}$. Since $\pi_{1} X$ is not a free group, $\pi_{1} M_{n}$ is not either.

Construct a tangle $Q_{n}=\left(B_{n}, A_{n}\right)=\left(B_{n}, a_{1} \cup \ldots \cup a_{n}\right)$ as in Figure 2, where $V_{k}$ is the tangle $T_{k}$ above when $k<n$, and $V_{n}=T_{n-1}$. Notice that if we replace $a_{1}$ by a trivial arc $a_{1}^{\prime}$ near $\partial B_{n}$ with $\partial a_{1}^{\prime}=a_{1}$, we get a trivial tangle $U_{n}=\left(B_{n}, A_{n}^{\prime}\right)=\left(B_{n}, a_{1}^{\prime} \cup a_{2} \cup \ldots \cup a_{n}\right)$.

\section{(Figure 2)}

Lemma 5 The tangle $\left(B_{n}, A_{n}-a_{i}\right)$ is equivalent to the trivial tangle $\left(B_{n}, A_{n}^{\prime}-a_{i}\right)$ for all $i \geq 2$.

Proof. This is obvious when $n=2$. In general we need to show that when removing $a_{i}$ from $B_{n}$, the arc $a_{1}$ can be isotoped into $a_{1}^{\prime}$ without intersecting the other $a_{j}$ 's $(j \neq 1, i)$.

We notice the following relation between $Q_{n}$ and $Q_{n-1}$ : Let $b_{1}, a_{3}, \ldots a_{n}$ be the arcs of $Q_{n-1}$, where $b_{1}$ is the one with endpoints on the left hand side of $B_{n-1}$. Replacing $b_{1}$ by two parallel $\operatorname{arcs} c_{1}, c_{2}$, we get the tangle $Q_{n}^{\prime}=\left(B_{n}^{\prime}, C_{n}\right)=\left(B_{n}^{\prime}, c_{1} \cup c_{2} \cup a_{3} \cup \ldots a_{n}\right)$ in Figure 2. So $Q_{n}$ is obtained by gluing the tangle $A$ in Figure 2 to the left hand side of $Q_{n}^{\prime}$.

By induction, after removing $a_{i}$ from $Q_{n}^{\prime}(i \geq 3)$, the $\operatorname{arcs} c_{1}, c_{2}$ are isotopic to some trivial arcs $c_{1}^{\prime}, c_{2}^{\prime}$ near the left hand side boundary of $B_{n}^{\prime}$. By another induction, it can be shown that $c_{1}^{\prime}, c_{2}^{\prime}$ may be chosen to have no intersection in the projection diagram, 
i.e. $\left(B_{n}^{\prime}, C_{n}-a_{i}\right)$ is a trivial tangle. So $a_{1}$ can be further isotoped off the tangle $V_{1}$, and becomes a trivial arc in $Q_{n}$. Also, since $c_{1}, c_{2}$ are parallel, there is a disk $D$ in $Q_{n}^{\prime}$ with $\partial D=c_{1} \cup c_{2} \cup \gamma_{1} \cup \gamma_{2}$, where $\gamma_{1}, \gamma_{2}$ are two small arcs on $\partial Q_{n}^{\prime}$ connecting the ends of $c_{1}$ to that of $c_{2}$, and $\operatorname{Int} D \cap a_{j}=\emptyset, j \neq 1$. Hence when deleting $a_{2}$ from $Q_{n}$, the arc $a_{1}$ can also be isotoped through $D$ so that it is disjoint from $Q_{n}^{\prime}$, and a further isotopy will modify it into a trivial arc near the left hand side of $B_{n}$.

Now let $\Gamma$ be a planar graph in $S^{3}$ with no free edges. We want to find another embedding of $\Gamma$ which is minimally knotted. If some component of $\Gamma$ has only one vertex, we can add a vertex to the middle of an edge in that component, dividing it into two edges. This will not affect any planarity, and a minimally knotted embedding of this new graph corresponds to a minimally knotted embedding of $\Gamma$. Therefore, without loss of generality, we may further make the following

Assumption: Every component of $\Gamma$ has at least two vertices and two edges.

Label the edges as $e_{1}, \ldots, e_{n}$, so that $e_{1}$ and $e_{n}$ have a common endpoint. Let $p_{i}$ be the middle point of $e_{i}$. Choose an arc $C$ embedded in $S^{3}$, so that $C$ passes through $p_{1}, \ldots, p_{n}$ successively with endpoints on $p_{1}$ and $p_{n}$, and has no other intersections with $\Gamma$. We consider $\Gamma \cup C$ as a graph in the natural way.

Lemma 6 If $\Gamma$ has no free edges, then $\Gamma \cup C$ is indecomposable.

Proof. Since $\partial C=\left\{p_{1}, p_{n}\right\}$ are midpoints of $e_{1}, e_{n}$. Since $e_{1}$ and $e_{n}$ have a common vertex, we can connect $p_{1}$ and $p_{n}$ by an arc in $e_{1} \cup e_{n}$ to get an embedded circle $J$ in $\Gamma \cup C$ containing $C$. Let $S$ be a decomposing sphere of $\Gamma \cup C$ containing a vertex $v_{0}$ of $\Gamma \cup C$, let $B_{1}, B_{2}$ be 3 -balls such that $B_{1} \cap B_{2}=S$. Since $J$ is a circle, it is contained in $B_{1}$, say. Therefore $(\Gamma \cup C) \cap B_{2}=\Gamma \cap B_{2}$ is a subset of $\Gamma$ since the vertices of $\Gamma \cup C$ lie in $\Gamma$.

If $v_{0}$ is a vertex of $\Gamma$, then $v_{0}$ is not a point of $C$, so $C$ is disjoint from any edge of $\Gamma$ in $B_{2}$, contradicting the construction of $C$. If $v_{0}$ is not a vertex of $\Gamma$, then it is the middle point of some edge $e$ of $\Gamma$ because these are the only vertices of $\Gamma \cup C$ which are not vertices of $\Gamma$. Since $C$ is disjoint from $\operatorname{Int} B_{2}$, there are no other edges in $B_{2}$. So if $e$ is not completely contained in $B_{2}$, then it is a free edge because the end of $e$ in $\operatorname{Int} B_{2}$ can not be incident to any other edges. This is impossible by the hypothesis of the lemma. If $e$ lies completely in $B_{2}$, then $e$ is a component of $\Gamma$ because $v_{0}$ is not a vertex of $\Gamma$. This contradicts the above assumption that every component of $\Gamma$ has at least two edges. 
Proof of Theorem 1: Choose an arc $C$ as above. Let $B=N(C)$ be a regular neighborhood of $C$, and let $b_{i}$ be the $\operatorname{arc} e_{i} \cap B$. Then $\left(B, b_{1} \cup \ldots b_{n}\right)$ is a trivial tangle. Recall the trivial tangle $U_{n}=\left(B_{n}, A_{n}^{\prime}\right)$ defined before Lemma 5 . Choose a homeomorphism $\varphi: B \rightarrow B_{n}$ so that $\varphi\left(b_{i}\right)=a_{i}$ for $i \geq 2$, and $\varphi\left(b_{1}\right)=a_{1}^{\prime}$. Replacing $b_{1}$ by $\varphi^{-1}\left(a_{1}\right)$, we get another embedding $G$ of $\Gamma$ in $S^{3}$. We want to show that $G$ is a minimally knotted embedding of $\Gamma$ in $S^{3}$.

Identity $(B, G \cap B)$ with $Q_{n}$ by the map $\varphi$. Denote by $e_{1}^{\prime}=\left(e_{1}-b_{1}\right) \cup a_{1}$ the edge in $G$ corresponding to $e_{1}$ in $\Gamma$. Clearly, $G-e_{1}^{\prime}=\Gamma-e_{1}$ is unknotted. Also, by Lemma 5 , when deleting any edge $e_{i}(i>1)$ from $G$, the edge $e_{1}^{\prime}$ can be isotoped to $e_{1}$, so $G-\operatorname{Int} e_{i}$ is isotopic to $\Gamma-\operatorname{Int} e_{i}$ in $S^{3}$ for all $i$. Hence any proper subgraph of $G$ is unknotted in $S^{3}$. It remains to show that $G$ is knotted.

Consider $E(G)=S^{3}-\operatorname{Int} N(G)$, the exterior of $G$. It suffices to show that $E(G)$ is not a handlebody. Recall the tangles $V_{i}$ in $B_{n}$, (see Figure 2). We use the same notation $V_{i}$ for the underlying 3-ball of $V_{i}$. Let $M_{i}$ be the manifold $V_{i} \cap E(G)$, and let $X=E(G)-\cup \operatorname{Int} M_{i}$. We have $E(G)=X \cup\left(\cup M_{i}\right)$. The surface $F_{i}=X \cap M_{i}$ is a punctured sphere, which by Lemma 4 is incompressible in $M_{i}$. We want to show that it is also incompressible in $X$.

Assume $F_{i}$ were compressible in $X$. Shrinking each 3-ball $V_{i}$ into a single vertex $v_{i}$, we get a new graph $G_{1}$. It is clear that $X=E\left(G_{1}\right)$, and the surface $F_{i}$ is exactly the punctured sphere $\delta\left(v_{i}\right)=\partial N\left(v_{i}\right) \cap E\left(G_{1}\right)$. By Lemma $1, F_{i}$ is compressible in $X$ if and only if $v_{i}$ is a decomposing vertex of $G_{1}$, so $G_{1}$ would be decomposable.

Denote by $G_{2}$ the graph obtained from $G_{1}$ by successively removing parallel edges of $G_{1}$ in the 3-ball $B_{n}$ until there are no parallel edges in $B_{n}$. The part of $G_{2}$ in $B_{n}$ is shown in Figure 3. There are $n-1$ cycles $r_{1}, \ldots, r_{n-1}$ in $G_{2}$, each of which bounds a disk $D_{i}$ in $B$ intersecting $G_{2}$ at a single point. Let $G_{3}$ be obtained from $G_{2}$ by shrinking every $D_{i}$ into a single vertex. By further deleting parallel edges of $G_{3}$ in $B$, we get a graph $G_{4}$. The part of $G_{4}$ in $B$ is shown in Figure 4 .

(Figure 3)

(Figure 4)

It is now clear that $G_{4}=\Gamma \cup C$. By Lemma 2 and Lemma 3, if $G_{1}$ is decomposable 
then so is $G_{4}$. On the other hand, Lemma 6 says that $\Gamma \cup C$ is indecomposable. This is a contradiction, therefore $F_{i}$ is incompressible in $X$.

The manifold $E(G)$ is the union of $X$ and the $M_{i}$ 's along the surfaces $F_{i}$. Since every $F_{i}$ is incompressible in both $M_{i}$ and $X, \pi_{1} M_{i}$ is a subgroup of $\pi_{1} E(G)$. By Lemma 4, $\pi_{1} M_{i}$ are not free groups, so $\pi_{1} E(G)$ is not a free group. But this certainly implies that $E(G)$ is not a handlebody, and so $G$ is a knotted graph.

Therefore, $G$ is a minimally knotted embedding of $\Gamma$.

\section{References}

[1] L. Kawauchi: Hyperbolic imitation of 3-manifolds, preprint (1987).

[2] J. Simon: Molecular graphs as topological objects in space, Journal of Computational Chemistry 8 (1987) $718-726$.

[3] J. Simon and K. Wolcott: Minimally knotted graphs in $S^{3}$, Topology and its Appl. 37 (1990) $163-180$.

Department of Mathematics,

University of California,

Santa Barbara, CA 93106

Current Address:

Department of Mathematics, University of Texas at Austin

Austin, TX 78712

E-main: wu@math.utexas.edu 